\title{
A Review of Virtual and Augmented Reality Concepts, Technologies and Application
}

\author{
Chris Duncan Lee \\ School of Computing and Data Science, Xiamen University Malaysia, Selangor Darul Ehsan, Malaysia. \\ duncanxiamen@outlook.com
}

\author{
Article Info \\ Journal of Computing and Natural Science (http://anapub.co.ke/journals/jcns/jcns.html) \\ Doi: https://doi.org/10.53759/181X/JCNS202101020 \\ Received 20 April 2021; Revised form 28 May 2021; Accepted 20 July 2021. \\ Available online 05 October 2021.
}

(C)2021 Published by AnaPub Publications.

\begin{abstract}
Consumers and academics are paying attention to affordable Virtual Reality (VR) (VR) remedies such as the Sony Entertainment VR, Vive VR and Oculus VR, including the Mixed-Reality Interface (MRITF) such as Hololens, which suggests it may be the next big thing in technical advancement. Nevertheless, VR has a wide history: the ideology of the remedy was initiated in 1960s, and commercialized toolkit for VR in the 1960s introduced in 1980s. This paper starts with the analysis of the development from VR to Augmented Reality (AR). We conclude by evaluating implications that MRITF, AR and VR will be capable of succeeding in the scientific disciplines, incorporating the human interaction as evident in the advent of cellular devices, altered social engagement, and understanding among individuals, as occurred with the emergence of smartphones.
\end{abstract}

Keywords - Virtual Reality (VR), Mixed Reality Interfaces (MRITF), Augmented Reality (AR).

\section{INTRODUCTION}

Thousands of scholarly publications have resulted from hundreds of researchers investigating the processes, impacts, and implementations of such an innovation during the past two decades. What is the result of this important scientific project? This article aims to address this issue by examining the current research corpus in the area using sophisticated scientometric methods. We compiled all of the available publications on Virtual Reality (VR) in the Science direct database, yielding a record with 21,668 VR items and 9,945 Augmented Reality (AR).

The present research looks at how Virtual Reality (VR) has evolved and changed through time in the major application segments, which key focus on the VR potential, developments, and key issues [1]. Within the past 5 years, VR and AR have stimulated the attention of the public and future stakeholders, most when the founder of Facebook purchased Oculus for about \$2.1 billion. Various firms, eg Microsoft are actively investing in virtual reality and augmented reality [2]. Despite VR, that has been used in research for more than twenty-five years and has hundreds of papers and a huge network of academics, AR is a comparatively new use.

Virtual reality research started in the realm of computer visuals and has since grown to encompass a wide range of disciplines. Virtual reality (VR) gaming is growing more mainstream than ever, and it is becoming an essential tool for brain research, psychologists, geology, and other scholars. Indeed, navigation studies are one of the study's main goals that integrate complex activities and experiments, which might be performed within a laboratory setting based on the application of VR, whereby scholars would have to get to the ecosystem potentially with limited assistance.

The relevance of route planning research for the properly functioning knowledge of human brain in alzheimer's disease has been a contentious issue for a long period of time. Moser were awarded the Nobel Prize in "Nobel prize in medicine" for their findings of neurones that facilitate a sense of place and route planning. Periodicals and publications have added to this understanding by reporting on "the cerebral GPS," which explains the process in detail. VR has been used in a large array of medical investigations.

K. Petri in [3] discussed the usage of VR, emphasizing its significance in both research and therapeutic practice. Furthermore, the range of available VR computational and experimental tools has made any area accessible. Augmented reality is a novel initiative compared Virtual reality, and it demonstrates a heterogeneous application model in which knowledge and education are the most studied area at the moment. In essence, AR might help educators by enhancing the comprehension of materials, learning motivations, and retention of memory. AR, on the other hand, is still developing in scientific situations, despite VR's clearer and more defined domains of application and study topics.

In this article, we add on the study of algorithmic and rigorous research concerning the development of interdisciplinary AR and VR scope based on the varied citation systems to assess the advancement of cognitive domain's smart structures throughout time. In that regard, this research paper has been arranged as follows: Section II concentrates on a survey of literature. Section III presents the methodology while Section IV focuses on a critical analysis of the theme. Section V evaluates the results and presents the relevant discussions. Lastly, Section VI concludes the research. 


\section{LITERATURE REVIEW}

I. Hughes in [4] conducted a research on citation networks and clusters in VR. Manuscript co-citation research is yet another research that can be conducted, which allows us to focus on most often citations in the sector, which seem to be the most impactful. Luisier, Yooyen and Deebhijarn in [5] evaluated the perceptions and functions of VR. In the 1960s, the phrase "virtual reality" to characterize windows wherein a user views the digital space as though it was real, and that users might behave realistically. Different formulations have been developed since that time, according on the application area.

VR was defined by C. Borst, J. Tiesel, and C. Best in [6] as "legitimate interactive visualizations utilizing threedimension frameworks integrated with the devices of presentation, which endorse the users to participate fully in the regular world and control it directly." The scholars also define VR as the impressions of engagement within the synthetic segment compared to passive observations on someone. VR utilizes a three-dimensional stereoscope tracker display, body and hand sensors, and stereo speakers. VR denotes to the multi-sensor, interactive, complete, viewer-based, threedimensional computer-based ecosystem and the integration of advancement required regenerating them.

According to T. Chung in [7], these criteria emphasize three similar characteristics of immersive technologies: absorption, sense of being there in a world, and engagement with that reality, despite their differences.

Immersion is defined as the stimulation of many senses, connections, as well as the similarities of realities to stimuli applied in the construction of virtual ecosystems. The characteristics of the technological structure employed to separate the user from reality may influence this attribute.

I. Heldal in [8] attest that three kinds of VR structures are available to the user, each with varying degrees of immersion: Non-immersive technologies, which utilize desktops to recreate pictures of the world, are the most basic and inexpensive VR applications; Immersion technology enables a variety of sensory external devices; including The Head Mounted Display (HMD) that develops the stereoscopic vision of the globe through locomotion, and sound and touch devices, to offer a full simulation session. Between the two, semi-immersive technologies like Fish Tank VR exist. They use a prospective projections combined with the viewer's body position to produce stereo images of a three-dimensional photography as visualized on a display. Higher-tech immersive technologies have provided users the impression of technical non-mediation and a sense of "to be in" or being in the virtual space. Additionally, more immersive structures allow for the inclusion of additional perceptual outputs, enabling interactions and activities to be experienced as genuine.

Lastly, by evaluating degrees of presence, realism, and actuality, the user's VR experience may be revealed [9]. Presence in VR is a deep psychological experience of "being in there" that includes the experience and impression of physicality, and the ability to communicate and respond as if the user were in the actual world. Consequently, the extent of anticipation the client has about the inputs and experiences is reflected in the realism degree. If the stimuli are authentic, the VR participant's thoughts will match reality, enhancing the virtual experience. In the same way, the more realistic the user's actions are, the more realistic the user's interactions with computerized stimuli are.

\section{METHODOLOGY}

The computed information and data were obtained from the keywords "AR" and "VR" from the WoS (Web of Science) database throughout a particular span (2009 to the present). The resulting collection included a record of 21,668 VR recordings and 9,945 AR recordings. Authors, titles, summary, and all citations were included in the reference list (needed for the citation analysis). Cite spaces Version 4.0 R5-SE-32 bits) was used to display the connections, and it ran on Java Runtime Version 8 update 91. StataMP Parallel Editions, Version 14.0, Stata Corp LP was used to perform statistical models. The degree to which a cluster in a networks is part of routes that link any two nodes in the network is measured by its closeness centrality. Betweenness centrality, diversity, and footprint are examples of structural measurements. Reference burstness and uniqueness are two distinct and composite metrics. All of the techniques are described in great depth.

\section{CRITICAL ANALYSIS}

\section{Virtual Reality (VR) (VR) to Augmented Reality (AR)}

Looking back at the history of VR and AR, the first 3D immersion simulation model was developed in the 1960s by researchers who produced Sensorama, a virtual sensation of a motorbike racing through Brooklyn that included auditory, olfactory, and haptic inputs, as well as wind to give a realistic simulation. During the same time period, Ivan Sutherland created The Ultimate Screen, which contained interactive visuals in addition to noise, odour, and sensory input, which Sensorama did not. In addition, Philco created the first head-mounted display (HMD) capable of updating simulated pictures through monitoring of the head directions and locations of the users utilized in linkage with the Damocles Sword of Sutherland. GROPE, the first force-feedback advanced technologies, was developed at the University of North Carolina, and Video-Place, a VR wherein viewers' musculature percentages were documented by camera systems and showcased on a screen, was developed by M. Krueger in [10].

This allows multiple users to effectively interact in two-dimensional simulated space. In the 80s, the Americal Air force established a simulated program, (VCASS - Visually-Coupled Airborne Structures Simulators) that permitted pilots to control the route and targets using a head-mounted display. The DataGlove, a synthetic rubber sensor that could sense finger flex, orientation, and placement, as well as recognize hand motions, was released in the late 1980s by the VPL Company. The VPL Company's Eyephone is another example of a head-mounted display (HMD) device that completely allows users into a virtualized ecosystem. Towards the end of 80s the Fake Space laboratory established the BOOM 
(Binocular Omni-Orientational Minitors, more complex system, which characterized the stereo devices, which provided a more dynamic and wider virtual ecosystem and a physical arm tracking.

Additionally, compared to HMD devices, BOOM provided a steadier picture and faster reactions to motions. The NASA Ames Research Facility created the Simulated Wind Tunnel with the help of DataGlove and BOOM to study and control aerodynamics in a digital aircraft or spacecraft. The CAVE Automated Virtual Environment, developed by the University of Illinois' Electronics Graphics Lab in early 1990s, is an immersive VR structure consisting of projections focused on 3 or more surfaces of a building. Many gaming firms have lately enhanced the social progress and better standards of VR instruments e.g. Oculus Rift, and HTCVive that provide massive vision field and minimized latency. Moreover, monitor systems such as eye tracking systems and orientation sensor might be incorporated within the actual HMD instrument - for instance, Razer Hydra. Concurrently, around the turn of the century, the Boing Corporation developed the first version of an AR structure to teach workers how to establish a wire tool.

S. Meža, Ž. Turk and M. Dolenc in [11] created an AR component for management help at the same time, demonstrating that adding digital environment on the component to fix improved operator productivity. The researchers developed an initiative for AR GPS advancement for evaluating blinds within the process of navigation. This program, the Spatial Audio Data was applied. E. Simão and P. Guerra in [12] evaluated the dance movement within the virtual spaces as an AR theatre whereby performers were capable to interact with the virtual items within the stipulated timeframe.

J. Greene in [13] created the first Mobile AR Structure (MARS) a few years down the line, which could add virtual data about touristy structures. Since then, many apps have been developed: scientists produced the AR-Quake, which is a mobile AR gaming that used the cellphone camera, the web, and Satellites to add details about the recipient's surroundings. Other AR applications e.g. SiteLens and ARToolkit were established in 2009 to effectively augment the actual user's surroundings with virtual information. D'Fusion, an AR structure for project design, was created by Total Interaction in 2011. Lastly, Google established the wearable technology in 2013 and Hololens in 2015 and their applicability have been verified in several fields.

Virtual Reality (VR) (VR)

Techniques for $V R$

The devices utilized within the virtual world play a fundamental role in the advancement of fundamental virtual interactions from a technology perspective. According to the research, data transmission devices may be differentiated. Device drivers, which may vary from a basic keyboard or mouse to a suit that captures finger motions or a monitor that captures postures, enable the users can interact with the digital world. More specifically, the keypad, joystick, touchpad, and gamepad are simple workstation input devices that enable the user to provide discrete and continuous instructions or motions to the surroundings.

Other inputs include bend-sensing mitts, which record hand movements, directions, and movement patterns, and pinch mittens, which identify arm movements, as well as monitors, which track the user's range of movement within the real world and possibly consider them within a simulated ecosystem. Contrarily, output signal permits users to visualize, touch, hear or taste something that is taking place within the virtualized ecosystem. As mentioned earlier, visual gadgets provide a wide array of choices, ranging from the most simple (a monitor screen) to the most comprehensive (a virtual reality headset) (CAVE, HMD, Helmets, and VR Glasses). Acoustic, transducer, and tactile output data may also trigger body feelings, culminating in a more genuine virtualized world - for instance, haptic instruments might influence users' touch pressure and perception frameworks.

\section{Use for VR}

Since its inception, VR has been utilized in a variety of areas, including gaming, weapons experience, building style, schooling, growth and social training, models of medical operations, aid to the elderly and psychiatric therapies. The main VR software pieces of evidence, such as strengths and weakness, in several scientific disciplines, such as scientific knowledge, professional training, strength and conditioning, fitness conditioning, as well as sociological phenomena, moral behavioural patterns, and might be utilized in other sectors, e.g. business, recreation, media, cooperation and conferences, were considered in the recent assessments of scholars. Another key research presented this year concentrated on VR in psychological health, demonstrating its effectiveness in evaluating and treating a variety of psychological illnesses such as anxiety, schizophrenic, trauma, and disordered eating.

There are many options for using VR as a stimulus, substituting actual stimuli and reproducing an experience, which might be impossible in the actual world with significant realism. This is the reason why VR is significantly applied in the research field concerning novel methods to apply psychological counseling or education, such as to phobia-related issues (agoraphobia, phobia to fly, etc.). Or, more simply, it is utilized to enhance conventional motor rehabilitative methods by creating games that increase task performance. In further detail, VR Exposure Therapy (VRET) has shown its effectiveness in psychiatric therapy, enabling patients to progressively confront fear stimuli or stressful circumstances in a secure setting where the therapist may regulate their mental and physiological responses. 
Augmented Reality $(A R)$

The Concept of $A R$

Researchers created the Immersive Spectrum, which considers four structures: the actual world, Augmented Reality (AR), enhanced digital world, and VR. AR is a novel technology and structure whereby virtual objects are integrated to an actual ecosystem in actual-time as the client is experiencing it. Researchers believe that an AR structure must: (1) integrate virtual and augmented items in a physical situation; (2) run dynamically and in instantaneously; and (3) associate physical and digital worlds with one another. Additionally, although AR encounters may seem to be distinct from VR structures, their quality may be compared. Furthermore, similar to VR, the sense of embodiment, immersion, and intensity of authenticity are the key characteristics that may be used to assess the quality of AR encounters. The greater the sense of "being there" physically, cognitively, and emotionally, the more genuine the experience is regarded. In both AR and VR settings, the sense of presence is critical for carrying out real-world activities.

\section{Technologies Using AR}

In terms of technology, all AR structures have three basic components: a geographical reference for virtual objects, e.g. virtual markers, a system to potentially display digitalized items to users, and sufficient computing capabilities for visuals, animations, and picture merging, such as a computer and a display. An AR structure needs include a camera that can follow the user's motions and combine virtual items with the real environment in order to function. A visual display, similar to glasses, that allows the user to view virtual things that are superimposed on the real environment.

Currently, there are two types of AR display structures: video see-through (VST) and optical see-through (OST). The first reveals digital environments to the user by using a camera to capture actual items/scenes and layering virtual elements, then displaying them on a video or display, while the second combines the digital objects onto such a transparent surface, such as eyeglasses, so the client could see the additional components. The distinction between the two technologies is bottleneck: an OST structure may take longer to show virtual elements than just a VST technology, resulting in a lag between both the user input and the program's recognition of them.

\section{Application of $A R$}

Irrespective of the ideology that AR is a novel initiate compared to VR, it has widely been researched in different segments such as psychiatric, clinical, recreation, educational, management, design therapies. In the field of education, several applications of AR have been created in recent years that demonstrate the potential of the technology in aiding learning, such as improved material comprehension and memory retention, and effective learning. For instance, they created an AR implementation for studying electromagnetic phenomena concepts, in which understudies might use AR battery packs, pieces of metal, and connectors on real surfaces, and the structure provided real-time feedback on the accuracy of their effectiveness, enhancing educational achievement and determination. In depth, the AR structure allows students to learn by seeing and acting on compound occurrences that were investigated conceptually but not seen or tested in real life.

The quantity of study on AR is growing, especially for the treatment of issues such as depression, much as it is in mental wellbeing. For instance, in the treatment of mental illness such as phobia, AR Exposure Therapy (ARET) shown effectiveness in a single session and continued to have a favorable effect at 1 or 3 months later. ARET, including VRET, creates a safe and ecological integrity in which any type of stimulus can be delivered, allowing patients to maintain control over the process while gradually yielding stress and fears. Actually, in cases of constant fears, such as irrational fears of animals, AR applications can progressively disclose the person to fear - inducing living creatures, introducing additional living creatures during the conversation, and increasing or decreasing the tempo in conformance with the participant's anxiety. Several studies have shown that AR can stimulate clients' depression at the start of a session and then reduce it after one hour of exposure. Patients were able to confront, communicate with, and kill authentic fear - inducing living creatures after the session, allowing them to effectively manage their anxiety and fear.

\section{RESULTS AND DISCUSSION}

An examination of the literature on VR reveals a complicated picture. At first glance, as per Web of Conferences (WoC) document-type statistical data, deliberations publications were widely used as study findings, accounting for nearly $48.5 \%$ of the sum (10,393 deliberations), with a comparable series of articles on the specific topic accounting for roughly $46 \%$ points of the combined capacity (10,200 editorials). Nevertheless, if we just look at the past 5 years (7,756 articles, or approximately $36 \%$ ), the picture theatrically changes, with publications accounting for around 56 percent of the total $(4,446)$ and proceedings accounting for about $34 \%$ as in D. Sirazhiden in [14]. As a result, it is evident that the VR sector has evolved in domains other than technology. Access points and interconnections are calculated as an occurring theme classes of the WoC "class" metric in various journals for the "classification" theme.

As per the WoS's topic category data, computer programming is the most popular, then technology and all these add to the 15, 342 journals. Nevertheless, if we just look at the past five years, these subcategories account for only approximately $56 \%$ of all articles, with a totaling of 4,283 publications.

The data is intriguing because it demonstrates that VR is a hot topic as a technological advances, with a lot of interest in both software and hardware components. However, in comparison to previous years, we are seeing an increase in the number of applications, particularly in the medical field. Its presence in the top ten lists of therapeutic and therapeutic neuroscience subcategories is very noteworthy (about $11 \%$ of the overall production in the past five years). It's also worth 
noting that, when taken together, neurobiology and psychiatry have grown from around $13 \%$ to nearly $17 \%$ in the past five years. Control and automation technologies, image science and photography science, and nanotechnology, which formerly amounts to approximately $15 \%$ of all articles published, were not in the top ten over the past five years, with each contributing for much less than $5 \%$.

As structures of founder nations, hubs and connections are calculated for each country. A country's numerous occurrences in the same article are only counted once.

Nearly half of the value was generated by the countries that were heavily involved in VR research $(10,200$ articles altogether). Out of a total of 10,202 papers, Germany, England, China, and the United States published 4920, 2385, 1498, and 1399 documents, correspondingly. The cases remain constant whenever we evaluate the scholarly publications from the last five years. Virtual reality donations, on the other contrary, happened to come from all around the world, with South Korea, Holland, Spain, France, Italy, Canada, and Japan leading the way.

With 160 publications from the University of Illinois, and 174 publications from the University of Washington and 148 publications from the University of South California, a global assumption on virtual reality was formed from published journals. Also from the United States was Iowa Public University, which was ranked ninth in the world (117). Canada came in second with 126 publications, with the University of Michigan in fifth place and Brown University in 10th position with 104.

Other nations in the list incorporated Holland, which was ranked fourth with 130 articles; Italy, which was the sixth with similar publication number and England ranked $7^{\text {th }}$ with 126 publications and 126 articles from Imperial College of Science at the University of London. Italy's Instituto de Auxologico Bello, which came in fifth place, was non-college group in the list for the research on VR. The highly ranked publications for VR citation are Tele Operators and Virtual Surrounding was 2690 publications and the Cyber Psychology and Behaviors with approximately 1885 references; Nonetheless, while the former has continued to increase citations in the last five years, the latter has enhanced by a far higher proportion, from about 70.5 percent to about 90.5 percent, or from 1030 to 1148 .

The two top publications, Institute of electrical and electronics Computer Animation and IEEEComput Graph, and StHEALT, were eliminated from the list centred on an assessment of the past 5 years. The list of three journals that outline the restorative classification and medical neuroscience, and neuroimaging and neuroscience, including both, included Exploratory Brain Science (750 citations), Archival materials of Rehabilitation Medicine (621 papers were identified), and Plos Person (620 papers were identified) [15].

Our findings are significant for 2 purposes. Initially, the latest research concentrated on the development and advancement of virtual and augmented reality research, providing clearer images centered on realistic data concerning a computer algorithm. Secondly, the assumptions on virtual reality revealed that the diagnostic and therapeutic component is among the most researches conducted in history, with both theoretical and practical elements continuing to increase, as well as advancement of technologies and publications in computer programming, engineering, and related disciplines.

Children's \& healthcare interface, frequent application and behavioral evaluation, special implications, structural perspectives and lessons were all part of the early stages of VR research. We may call the development era the pioneering age since VR was applied in laboratory experiments that related to novel technological impulse. It was also during the late 1990s, when the so-called "dot-com ripple" burst, coinciding with novel economic generation, when massive investments in computer technology were made. Using the confluence of groundbreaking methodologies into ergonomic research, the first effective therapeutic techniques for operations, telemonitoring, cognitive spatial awareness, and the beginning of the evolution of diagnosis and laparotomy skills were developed during this development period. With a decrease in recidivism, neurophysiology, and a new coalition of restorative and arthroscopic skills, VR research dramatically shifted toward what we might call the clinical-VR period around the beginning of the century. The upsurge in the numbers of cellular application and journal presented in the past five years coincides to new scientific advancement on both the hardware and software levels, with a growing number of independent development companies and VR connections.

Lastly, the dynamics of identifying the origin, present, and prospective of AR studies are not yet apparent, but current fields of AR study include examining the connections between these groupings and the temporal characteristics of every article analytics, educational, and the digital city context. AR is a revolutionary innovation that is demonstrating its effectiveness in a variety of areas, as well as offering a unique method to collect physiological information to assist learning, education, and therapeutic therapies.

\section{CONCLUSION}

Although the ramifications for patients are enormous, it is apparent from this study that the potential of Virtual and AR study is not only in therapeutic applications. Virtual Reality (VR) and Augmented Reality (AR) structures are constantly evolving as a consequence of study in computer science, economics, and other related fields. There are three causes for the emergence of a "clinical era" as a result of our research. To begin with, every medical study on VR and AR involves technical advancements, and innovative technical findings are posted in medical or mechanical publications, but with clinical specimens as the primary focus. Researchers in psychoanalytic theory, neuroimaging, healthcare, and cognitive science in particular have clearly been continuing to investigate if the advances in technology of Virtual and AR are beneficial for users, implying that clinical cognitive psychologists has incorporated large parts of engineering and computer science. The industrialization of the country is a second factor to consider. In reality, once a new invention has been conceived and developed, it is filed for a patent. The new innovation may be made available for sale once the 
copyright has been registered, and eventually for submissions to publications. Moreover, AR and VR studies that showcase a structure's advancement jump from showcasing prototypes to getting patents and supplying it to the market without considering a release of the results in the scholastic journals. Therefore, it goes without saying that if new software is implemented for the commercial or service sectors and not for therapeutic use, the research that went into developing it will almost likely never be published in a scholarly journal. Notwithstanding the fact that this article cantered on previous papers, we must acknowledge the existence of many unpublished studies. The fact that numerous articles on VR and AR were analysed in the Web of Knowledge repository, which really is our repository of citations, is the third explanation for our results alluding to a "medical era." The dataset we were looking at was referred to as "research" in this article. This is a restriction in our research, as there is a slew of databases, which are vital in the scholastic societies, e.g. IEEE Xplore Digital Collection, Electronic Databases, and a slew of others. In general, the Web of Knowledge database contains the most important articles in academic journals in these repositories; therefore, we conclude that our research integrates the most fundamental research in computer science and engineering. As a result, we think that by considering the vast number of papers cited in our study, this restriction may be addressed. Taking all these factors into account, it's obvious that therapeutic options, behavioural elements, and technical advances in Virtual and AR studies are all part of a more complicated scenario than the previous platforms that existed before to the widespread use of HMD and services. We believe that our effort will provide consumers a better picture of current research frontiers and future problems, emphasizing all the linkages and consequences of research in a variety of areas, including medical, psychological, industrial, recreational, pedagogical etc.

\section{References}

[1]. A. Riener, J. Gabbard and M. Trivedi, "Special Issue of Presence: Virtual and AR Virtual and AR for Autonomous Driving and Intelligent Vehicles: Guest Editors' Introduction", PRESENCE: Virtual and AR, vol. 27, no. 1, p. i-iv, 2019. Doi: 10.1162/pres_e_00323.

[2]. J. Park and S. No, "A Study on the Structural Features of VR HMD Interface Design - Focused Oculus Home -", Journal Of The Korean Society Design Culture, vol. 24, no. 3, pp. 281-296, 2018. Doi: 10.18208/ksdc.2018.24.3.281.

[3]. K. Petri, "Towards the Usage of VR for Training in Sports", Biomedical Journal of Scientific \& Technical Research, vol. 7, no. 1, 2018. Doi: 10.26717/bjstr.2018.07.001453.

[4]. I. Hughes, "Virtual worlds, AR, blended reality", Computer Networks, vol. 56, no. 18, pp. 3879-3885, 2012. Doi: 10.1016/j.comnet.2012.09.016.

[5]. Luisier, Yooyen and Deebhijarn, "Perceptions of Thai Aviation Students on Consumer Grade VR Flight Experiences", Proceedings, vol. 39, no. 1, p. 8, 2019. Doi: 10.3390/proceedings2019039008.

[6]. C. Borst, J. Tiesel and C. Best, "Real-Time Rendering Method and Performance Evaluation of Composable 3D Lenses for Interactive VR", IEEE Transactions on Visualization and Computer Graphics, vol. 16, no. 3, pp. 394-410, 2010. Doi: 10.1109/tvcg.2009.89.

[7]. T. Chung, "A Study of Immersive Display Technologies", The Smart Computing Review, 2012. Doi: 10.6029/smartcr.2012.03.006.

[8]. I. Heldal, "Supporting participation in planning new roads by using VR structures", VR, vol. 11, no. 2-3, pp. 145-159, 2007. Doi: 10.1007/s10055-006-0061-3.

[9]. L. Daniela and M. Lytras, "Editorial: themed issue on enhanced educational experience in virtual and AR", VR, vol. 23, no. 4, pp. 325-327, 2019. Doi: 10.1007/s10055-019-00383-z.

[10]. M. Krueger, ""VIDEOPLACE": A Report from the Artificial Reality Laboratory", Leonardo, vol. 18, no. 3, p. 145, 1985. Doi $10.2307 / 1578043$

[11]. S. Meža, Ž. Turk and M. Dolenc, "Component based engineering of a mobile BIM-based AR structure", Automation in Construction, vol. 42, pp. 1-12, 2014. Doi: 10.1016/j.autcon.2014.02.011.

[12]. E. Simão and P. Guerra, "Virtual Dance Floors: Shall We Dance or Fly?", Dancecult, vol. 12, no. 1, 2020. Doi: 10.12801/19475403.2020.12.01.04.

[13]. J. Greene, "Creating Mobile AR Experiences in Unity", The Programming Historian, no. 7, 2018. Doi: 10.46430/phen0073.

[14]. D. Sirazhiden, "VR and AR technologies in the modern cultural space and their role in environmental education", E3S Web of Conferences, vol. 217, p. 08002, 2020. Doi: 10.1051/e3sconf/202021708002.

[15]. E. Ch'ng, "Living Virtual Heritage: Agents and Enhanced Environments", Presence: Teleoperators and Virtual Environments, vol. 23, no. 3 , pp. 339-339, 2014. Doi: 10.1162/pres_x_00200. 